\title{
Los servicios de los ecosistemas de Chocontá: un ejercicio de percepción con sus habitantes
}

\author{
Maritza Duque Gutiérrez', Carlos Montes del Olmo² \\ 1. Docente Universidad Santo Tomás. Facultad de Ciencia y Tecnología. \\ Programa de Administración Ambiental y de los Recursos Naturales. \\ 2. Director Laboratorio de Socioecosistemas. Facultad de Ciencias. Universidad Autónoma de Madrid. \\ Campus de Cantoblanco 28049. Madrid (España).
}

Correspondencia: maritzaduque@ustadistancia.edu.co

Recibido: 14-10-2011 / Aceptado: 30-12-2011

\begin{abstract}
Resumen
El presente artículo propone una nueva visión sobre el territorio del municipio de Chocontá, Cundinamarca (Colombia), al hacer una aproximación desde la economía ecológica, en la que, los componentes social, económico y cultural, configuran un subsistema del sistema ecológico. En este municipio, la biodiversidad de los tres ecosistemas de rango menor que contiene, genera diversos eco-servicios que contribuyen al bienestar de sus habitantes. Con el fin de caracterizar la identificación de los servicios que genera dicha biodiversidad se indagó la percepción de sobre éstos a diferentes actores sociales del territorio mencionado. Los resultados muestran los estrechos vínculos entre el bienestar humano y el capital natural, los cuales por procesos de globalización económica, han venido en detrimento.

Palabras clave: economía ecológica, biodiversidad, eco-servicios, bienestar, actores sociales, capital natural.
\end{abstract}

\begin{abstract}
The ecosystem services of Chocontá: an exercise of perception with their inhabitants

This article proposes a new vision for the municipality of Chocontá, Cundinamarca (Colombia), to make an approach from ecological economics, in which, the social, economic and cultural make up a subsystem of the ecological system. In this municipality, the biodiversity of the three lower-ranking ecosystems it contains, generates various eco-services that contribute to the welfare of its inhabitants To characterize the identification of the services that biodiversity generates, were investigated on the perception of these different social actors of the mentioned territory. The results show the close links between human welfare and natural capital, which by processes of economic globalization, have been detrimental.
\end{abstract}

Key words: ecological economics, biodiversity, ecosystem services, welfare, social actors, natural capital.

\section{Introducción}

Los servicios de los ecosistemas (ecoservicios), son los beneficios que las personas obtienen de los ecosistemas, que hacen la vida humana físicamente posible y digna de ser vivida (1). En general se consideran tres categorías de servicios: abastecimiento, regulación y culturales $(2,3)$.

En otra manera de decirlo, los ecoservicios son los beneficios que las comunidades humanas reciben a partir de los procesos, funciones e 
interacciones armónicas con los ecosistemas. Tales como abastecimiento o regulación y culturales.

Los servicios de abastecimiento son aquellos servicios recibidos directamente de la estructura de los ecosistemas y que contribuyen al bienestar humano. El principal servicio de abastecimiento de Chocontá, es el alimento, proveniente de la cría de animales, los cultivos y la recolección de frutos silvestres. Otros servicios de abastecimiento son los materiales de la explotación de canteras y el suministro de agua para consumo.

Los servicios de regulación son aquellos beneficios recibidos del funcionamiento de los ecosistemas, es decir aquellos procesos necesarios para la generación de servicios de abastecimiento como el ciclo hidrológico o la fertilización del suelo.

Los servicios culturales hacen referencia a los servicios intangibles que ofrecen los ecosistemas y están íntimamente ligados a los rasgos culturales, morales y éticos de la sociedad. Dentro de estos están la identidad, la recreación y el ecoturismo, la educación ambiental y el conocimiento científico.

Lo anterior implica una relación de pares entre los componentes del ecosistema y la comunidad de seres humanos. En otras palabras, es redimensionar el carácter antropocéntrico y predador que las sociedades han tenido con los ecosistemas, de los cuales ella misma hace parte.

Esto quiere decir, que al hablar de comunidad (común-unidad) se hace referencia a la comprensión en términos de acción, palabra y pensamiento, de la unidad entre los seres humanos y los demás seres vivos, en el conjunto de interacciones vitales que entrańa un ecosistema. Lo anterior, implica unas responsabilidades humanas en el ofrecer a los demás componentes del ecosistema, unas acciones que contribuyan a su equilibrio y conservación, en medio de las transformaciones adaptativas de éste, como un gran organismo vivo.

En este trabajo no solo se han caracterizado los ecoservicios, sino también las funciones o procesos biofísicos esenciales que determinan la capacidad de generarlos, entendiéndose por estas la capacidad que tienen los ecosistemas de suministrar servicios para el bienestar humano (4) y son independientes de su uso, demanda, disfrute o valoración (5). Las funciones se clasifican en cuatro categorías que fueron empleadas para Chocontá:

- Funciones de regulación o la capacidad de los ecosistemas de regular los procesos esenciales de los ecosistemas. Por ejemplo: regulación hídrica, atmosférica y climática.

- Funciones de hábitat que se refiere a la oferta de condiciones espaciales los espaciales para la conservación de la biodiversidad. Por ejemplo: función de refugio y criaderos.

- Funciones de producción o la capacidad de los ecosistemas para proveer bienes para uso humano. Por ejemplo: alimento, materias primas y recursos genéticos.

- Funciones de información que ofrecen posibilidades para la reflexión, el enriquecimiento espiritual y el desarrollo cognitivo. Por ejemplo: Información estética, artística, cultural e histórica Para la caracterización de un territorio desde la integración de su sistema ecológico y social, las consideraciones anteriormente mencionadas constituyen un marco de análisis integral e integrador en el análisis de las relaciones naturaleza-sociedad. Por tanto en necesario el análisis de las componentes socioeconómicas y culturales y ecológicas en un marco unificador. En previas investigaciones (6) se realizó una caracterización de la Sabana de Bogotá como un sistema socioecológico (SES), donde quedó de manifiesto que uno de los municipios de mayor biodiversidad, también presenta características de necesidades básica insatisfechas altas.

Por lo tanto, el objetivo principal de esta investigación ha sido identificar, a través de encuestas de percepción, en cuáles elementos radica el bienestar de los habitantes del municipio de Chocontá. Lo anterior, en relación con los servicios que ofrecen sus ecosistemas, identificando los servicios que proporcionan la biodiversidad de Chocontá y las relaciones de los ecosistemas con el bienestar de sus habitantes.

\section{Materiales y métodos}

La identificación de los servicios que ofrecen los ecosistemas de Chocontá se realizó a partir de entrevistas y cuestionarios aplicados a la población, en forma aleatoria simple, en los que se indagaba, (a) la 
identificación de los ecosistemas presentes con el flujo de servicios, categorías y sub-categorías que generan cada uno y (b) la identificación de los beneficiarios de los servicios y (c) la escala en la que se reciben el bienestar. Se trabajaron encuestas estructuradas que se desarrollaron a manera de entrevista, para facilitar la comprensión de la información solicitada, por parte de los indagados las que se acompañaron de listados que comprendían los eco-servicios más importantes.

\section{Resultados}

En primer lugar, previo al análisis se identificaron los servicios que generaban cada uno de los ecosistemas presentes en el municipio, los que fueron clasificados también en categorías y subcategorías, Tabla 1.

\section{Los ecosistemas Chocontá y su importante flujo} de servicios. No existe una gran diferencia entre la cantidad de ecoservicios que ofrecen cada uno de los ecosistemas que conforma Chocontá, aunque el mayor porcentaje de suministro lo tiene el ecosistema del bosque andino bajo. Este ecosistema es el de mayor extensión en el municipio. En los otros dos ecosistemas, Bosque Altoandino y Subpáramo, los ecoservicios que más se presentan son los de abastecimiento, seguidos por los de regulación Figura 1.

\section{Descripción general de los principales ecoservicios} de Chocontà. A continuación se describen algunos servicios generados por los ecosistemas de Chocontá por tener mayor importancia y haber presentado cambios relevantes en la zona de estudio:

\section{Servicios de abastecimiento}

Alimentos. El indicador de este servicio es el número de hectáreas sembradas. El encargado de registrar esta información es el Instituto Colombiano Agropecuario. Los principales cultivos de Chocontá son papa, hortalizas y flores, en los cuales se mantiene un tipo de agricultura convencional, con muy poca área destinada a la producción ecológica. Los beneficiarios de este servicio es la población a nivel local y nacional.

El segundo mayor cultivo son las flores bajo invernadero, la especie más sembrada es la rosa (32\%),

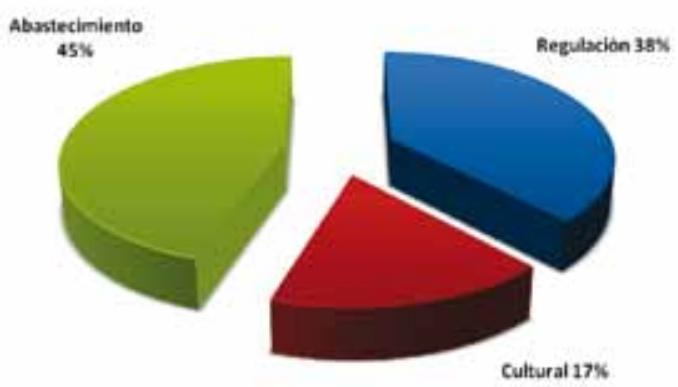

Bosque Alto Andino

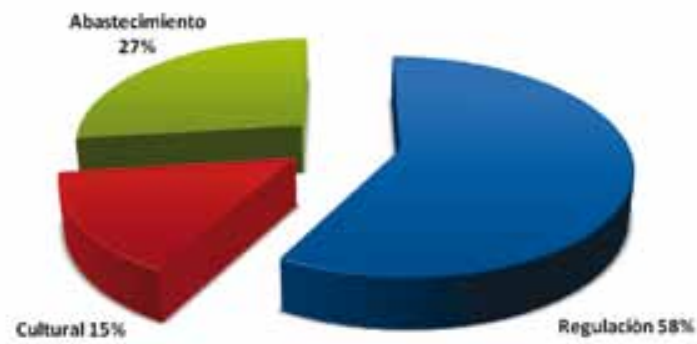

Bosque Andino Bajo

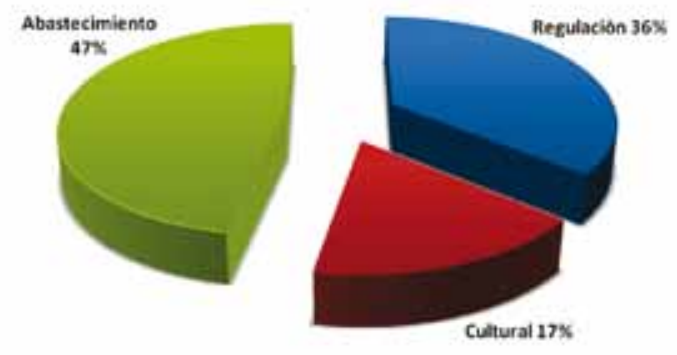

Subpáramo

Figura 1. Ecosistemas de escala menor presentes en Chocontá con el porcentaje de ecoservicios generados en cada uno de ellos.

seguido del clavel (14\%). Es decir, que los beneficiarios de este servicio están a escala internacional, siendo el mayor comprador Estados Unidos que recibe el 78\% de la flor producida en Colombia (8). El censo hortícola de 2002 (7) reporta producción de acelga, apio, brócoli, cilantro, coliflor, espinaca, lechuga, coliflor, remolacha, repollo y zanahoria. En general del área destinada a hortalizas se siembra el $22.7 \%$ en lechuga (383 ha), el $16.4 \%$ en zanahoria (277 ha), el $10.7 \%$ en repollo (188 ha) y el $9.2 \%$ en remolacha (155 ha). Aunque no se encuentra información detallada sobre el tamaño de los predios dedicado a cada hortaliza, el censo de hortalizas muestra que el promedio el 20.5\% del área de los 
Tabla 1. Principales ecoservicios y funciones identificados en el municipio de Chocontá.

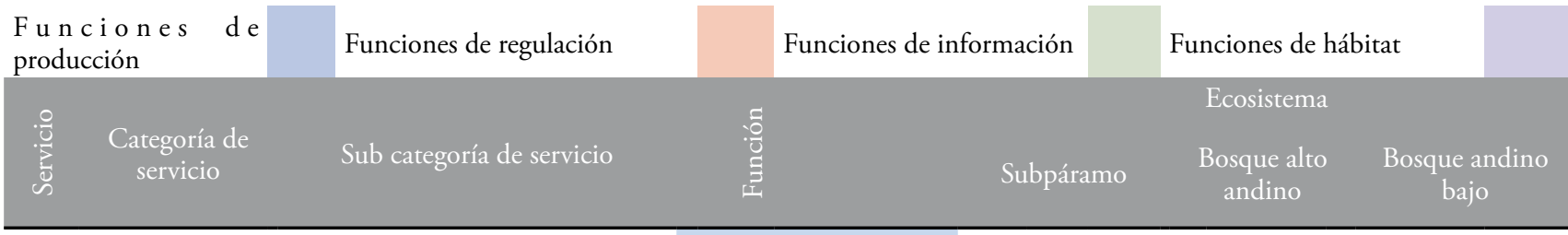

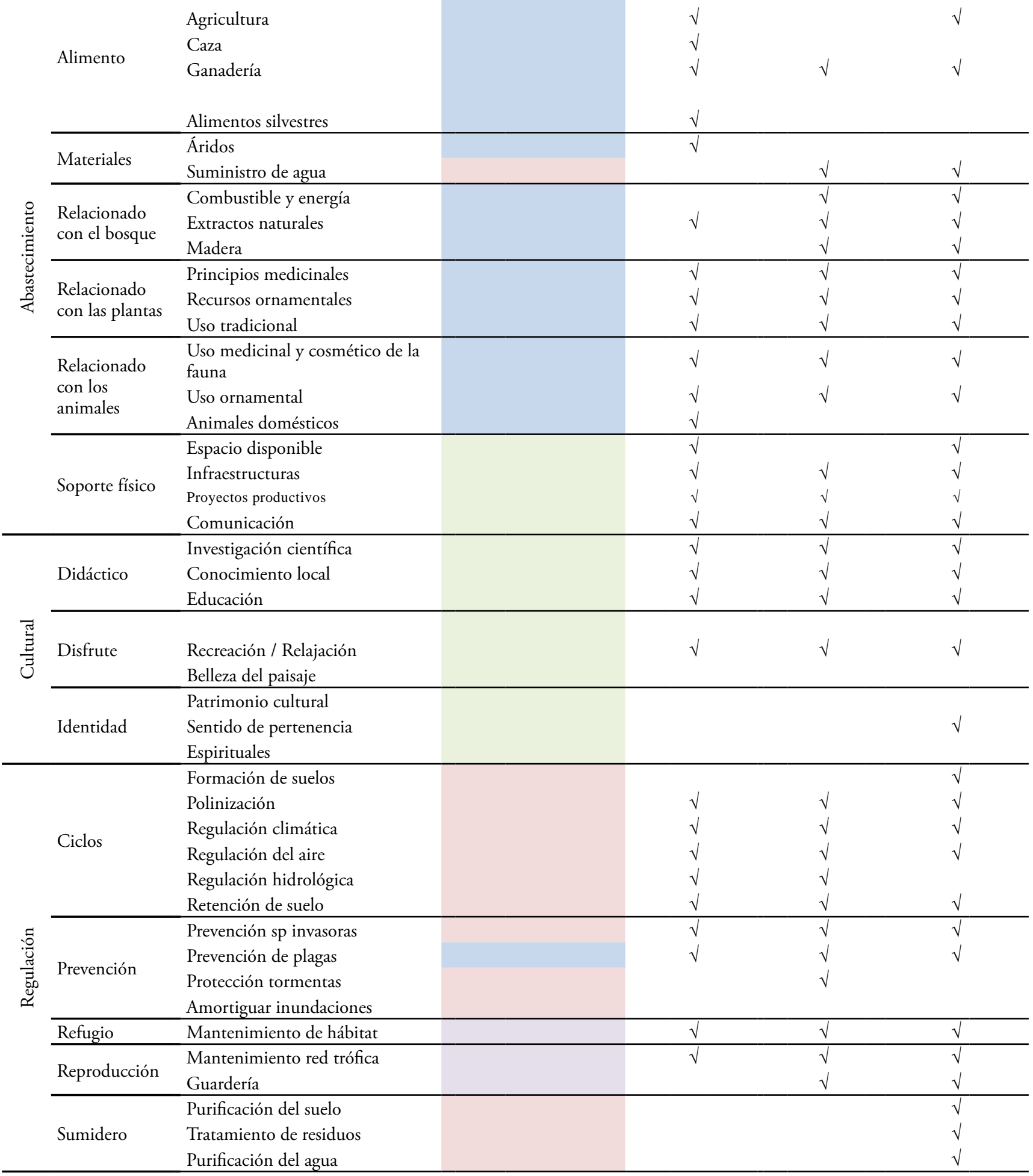


predios se siembra en hortalizas (9). De este servicio se beneficia la población a nivel local y nacional.

La ganadería está básicamente comprendida por ganadería bovina de leche. Los beneficiarios de este servicio se presentan a nivel local y departamental.

\section{Servicios de regulación}

Regulación hídrica. La conjunción del clima y las características de vegetación y suelos hace que algunos ecosistemas constituyan unos reguladores naturales de la escorrentía, como es el caso del páramo. Otros le asocian el efecto de esponja a la regulación hídrica, mostrando su importancia en los flujos en épocas de estiaje. En Colombia los páramos regulan el $70 \%$ de los ríos en sus cabeceras, aportan el $4 \%$ de la oferta hídrica superficial colombiana $(10,11)$ y un porcentaje importante de municipios andinos se abastecen de agua de estas fuentes (aproximadamente 60\% de los municipios de la SB) (12). Actualmente la producción agropecuaria se está presentando en estos ecosistemas. La quema de la vegetación de páramo hace que la materia muerta desaparezca y aparezcan nuevos brotes, esta vegetación fresca es atractiva para el ganado. El pastoreo causa la compactación del suelo. Por otro lado, la quema acelera el proceso de descomposición y genera suelos más secos incrementando los niveles de temperaturas (13).

Purificación del agua. Este servicio, en relación con el anterior, está siendo afectado por las actividades agropecuarias, debido a su explotación incontrolada y a la carga de metales pesados que recibe de los productos de síntesis química usados en estas actividades. Las curtiembres, localizadas en la cuenca alta del río Bogotá, en los municipios de Villapinzón y Chocontá, que vierten sus desechos al río han aumentado la carga orgánica (14).

Prevención de especies invasoras. El Instituto de Investigación de Recursos Biológicos Alexander Von Humboldt publicó la cartilla denominada: "Especies Invasoras Colombianas", en la cual se definieron cinco especies de plantas, cuatro especies de invertebrados, una especie de anfibios y tres especies de peces, como especies invasoras presentes en el territorio nacional, incluyéndo las especies Retamo espinoso (Ulex europaeus) y Retamo liso (Teline mons- pessulana) que tienen mayor presencia en Chocontá. En la actualidad algunos viveros y distribuidores de semillas ubicados en el territorio producen y comercializan plántulas y semillas de estas especies, siendo habitual, además, su uso como cerca viva y esto conjugado con la alta capacidad de dispersión de la especie podemos concluir que la prevención de especies invasoras se ha visto en declive.

\section{Servicios culturales}

Conocimiento local ecológico. Debido a la aptitud agrícola de Chocontá, el conocimiento ecológico tradicional ha jugado un papel importante en torno a la cultura campesina, no obstante se ha presentado una fuerte perdida en la transmisión intergeneracional, debido al cambio de actividades de las personas jóvenes, al no encontrar en las actividades del campo una buena alternativa de vida (15).

Belleza del paisaje. El paisaje se caracteriza por su clima frío y al mismo tiempo soleado y lluvioso, con contrastes entre la llanura y los cerros. Tomas Rueda insistía en que era un paisaje para observar y meditar y que esa función había sido fundamental en la caracterización de la clase dirigente bogotana. (16). Pese a la peculiaridad de sus ecosistemas, la "plastificación” de Chocontá (invernaderos de flores), la urbanización y el flujo industrial desde la capital, han generado detrimento en este servicio.

\section{La percepción de los servicios por parte de los actores sociales del municipio}

Las encuestas se realizaron entre los meses de marzo y junio de 2011 a diferentes actores del municipio, estas fueron acompañadas de listados de ecoservicios que permitía una orientación a los encuestados. Se presentan los resultados más relevantes:

1. Con respecto a la identificación de servicios, los de regulación, son los que las personas identifican como de mayor importancia, dentro de los que sobresalen los alimentos provenientes de agricultura y de ganadería.

2. Frente a la frecuencia de uso de los servicios, fueron clasificados como de uso diario y espacialmente son usados dentro del municipio.

3. Cuando se pidió a los entrevistados describir su municipio, lo relacionaron en su mayoria con 
un servicio cultural. Frente a la pregunta de qué le hace feliz, la respuesta estuvo orientada a servicios relacionados con la agricultura. En su mayoría servicios de producción de alimento (abastecimiento) y en segundo lugar a la relaciòn con los demàs (culturales). Cuando se mostraron los listados orientadores, la respuesta, en su mayorìa, estuvo relacionada con los servicios culturales, donde se resalta el turismo ecológico, la estética del paisaje y el turismo ecológico.

4. A la opción de identificar los hobbies, estos se relacionan con el deporte, lo que indica una estrecha relación con los servicios culturales.

5. Los entrevistados identifican a la contaminaciòn, la inseguridad y las basuras como los principales problemas del municipio, a lo que poponen soluciones de campañas de concienciación, aumento de vigilancia y control, y programas de manejo de residuos, respectivamente.

6. Con el uso de los listados a la pregunta de los servicios más vulnerables, en primer lugar esta al aire limpio (afectado por la mala calidad del transporte), la alteración de los paisajes (por la urbanización y la minería), la conservación de las especies (por la caza incontrolada) y el hábitat de las especies (a causa de la deforestación para minería).

7. Para el municipio se identificaron 5 figuras de protección y gestión del capital natural: Parque Nacional Natural, Bosque Municipal, Área histórico-cultural-Refugio de vida silvestre y Red de reservas de la sociedad civil. Pero en un los habitantes desconocen la presencia de estas figuras dentro del municipio, lo que muestra un claro deterioro del capital social, por la falta de identidad y desconocimiento del territorio

\section{Discusión}

Al realizar una visión histórica sobre los servicios que han generado los ecosistemas de Chocontá, se observa una merma y reducción de calidad de los servicios de regulación y de los servicios de abastecimiento en lo que respecta a la alimentación de la población del municipio. Pero aquellos servicios de abastecimiento relacionados a mercados externos como la papa, o fuera del país como las flores, han aumentado.
En la actualidad la gestión de este municipio se encuentra enfocada en pocos servicios. Por un lado a aquellos que buscan satisfacer necesidades externas y por otra el declive del conocimiento ecológico tradicional, generándose un aumento en servicios culturales nuevos como el ecoturismo. Tal demostró Bennett et al. (17), cuando la gestión se focaliza en unos pocos servicios, los trade-offs generados entre ellos pueden producir resultados no deseados como el declive de los servicios de regulación.

El modelo de gestión actual de Chocontá lleva a la degradación de servicios de regulación que son necesarios para el funcionamiento del ecosistema y la expresión de los servicios de abastecimiento y culturales, haciendo cada vez más vulnerable a los socioecosistemas para su recuperación después de una perturbación.

Aquellos servicios que llevan a satisfacer las necesidades de habitantes de fuera de Chocontá o internacionales como en el caso de las flores, tiene efectos negativos sobre el bienestar de los habitantes de Chocontá por la afectación a los servicios de regulación, relacionados al aire, agua y ciclaje de nutrientes.

Por otro lado, las encuestas de percepción muestran como las actividades tradicionales se ha ido perdiendo, reduciendo así la capacidad de sus habitantes de abrir su abanico de oportunidades laborales.

Poniendo de manifiesto las estrechas relaciones existentes entre el bienestar de los pobladores y la calidad de los ecosistemas permitirá tumbar barreras y tender puentes entre la gestión, la conservación y el bienestar.

\section{Agradecimientos}

Esta investigación ha sido financiada por el centro de Investigaciones de la Universidad Santo Tomás modalidad a distancia. Agradecemos al grupo de Investigación "Resilientes" por su apoyo en campo.

\section{Referencias}

1. Díaz S, Fargione J, Chapin F, Tilman D. Biodiversity loss threatens human well-being, PLoS Biology, 2006; 4:1300-1305

2. MA, millennium ecosystem assessment. (2003). Ecosystems and human well-being: A framework for assessment, Island 
Press, Washington, D.C. USA. 212 pp. URL: http://www.millenniumassesment.org/en/Framework.aspx

3. Carpenter R, Mooney H, Agard J, Capistrano D, DeFries R, Díaz S, et. Al. Science for managing ecosystem services: Beyond the Millennium Ecosystem Assessment. PNAS 2009;106: 1305-1312.

4. De Groot R, Fisher B, Christie, M y et al. (2010). The economics of valuing ecosystem services and biodiversity. En: Kumar, P. (Ed.), The Economics of Ecosystems and Biodiversity: Ecological and Economic Foundations. Earthscan, Londres

5. Martín-López B., E. Gómez-Baggethun, J.A. González, P.L. Lomas, C. Montes. 2009a. The Assessment of Ecosystem Services Provided by Biodiversity: Re-Thinking Concepts and Research Needs. En: Handbook of Nature Conservation. Aronoff J.B. (Ed). Nova Science Publishers, Inc.

6. Duque, M. 2010. Gestionando el capital natural de la Sabana de Bogotá para el bienestar humano: una aproximación a su concepción como socioecosistema. Universidad Internacional de Andalucía. 2010

7. Departamento Administrativo Nacional de Estadística (DANE), 2002. Sabana de Bogotá. Censo hortícola 2002. URL: http:// www.dane.gov.co/files/investigaciones/agropecuario/ena/ Censo Horticola Bta 2002.pdf

8. Asocolflores (2009). Colombian floriculture. Hoja de datos de la floricultura colombiana. URL: http://www.asocolflores.org/

9. Universidad Nacional de Colombia, SENA, Corpoica (2006). Corredor tecnológico agropecuario para la Sabana Occidente. Bogotá, 22 pp. URL: http://www.unal.edu.co/viceinvestigacion/ ct/corredoPlanestrategico.pdf

10. Cepis 2005. url: http://www.cepis.ops.oms.org/eswww/fulltext/ analisis/colombias/spcapit10.html
11. Ministerio del Medio Ambiente, 2005. "Lineamientos de Política para el manejo integral el agua”. http://www.col.opsoms.org/diia/2002/MINAMB lineamientos.htm\#775

12. Ministerio del Medio Ambiente, 2001. Programa para el manejo sostenible y restauración de ecosistemas de alta montaña: páramos

13. Domínguez, D. (1999). Hidrología de Páramos, Modelación de la cuenca alta del río Blanco. Universidad de Los Andes, Departamento de Ingeniería Civil y Ambiental. Bogotá

14. Jiménez AC, Cortés C E. "Cromo y sus compuestos”. En: "Enfermedades Profesionales: protocolos para su diagnóstico". Ministerio de Trabajo y Seguridad Social. Sociedad Colombiana de Medicina del Trabajo. Bogotá, Colombia. 1998

15. Alcaldía Municipal de Chocontá, 2011. Informe de gestión para la rendición pública de cuentas sobre la garantía de los derechos de las niñas, los niños, los adolescentes y los jóvenes. URL: http://choconta-cundinamarca.gov.co/apc-aa-fi les/38613735626339353361653063373163/INFORMEGESTIONINFANCIA.pdf

16. Umaña J. (2007). La Sabana de Bogotá y los ecosistemas relacionados en el 2007. (2007) FESCOL. URL: http://www. fescol.org.co/DocPdf/EV.FNA-SabanaBogotaEcosistemasRelacionados.pdf

17. Bennett E Peterson G, Gordon L. 2009. Understanding relationships among multiple ecosystem services. Ecology Letters, 12: $1394-1404$.

18. Gordon L, Finlayson C, Falkenmark M. 2010. Managing water in agriculture for food production and other ecosystem services. Agricultural Water Management, 97: 512-519.
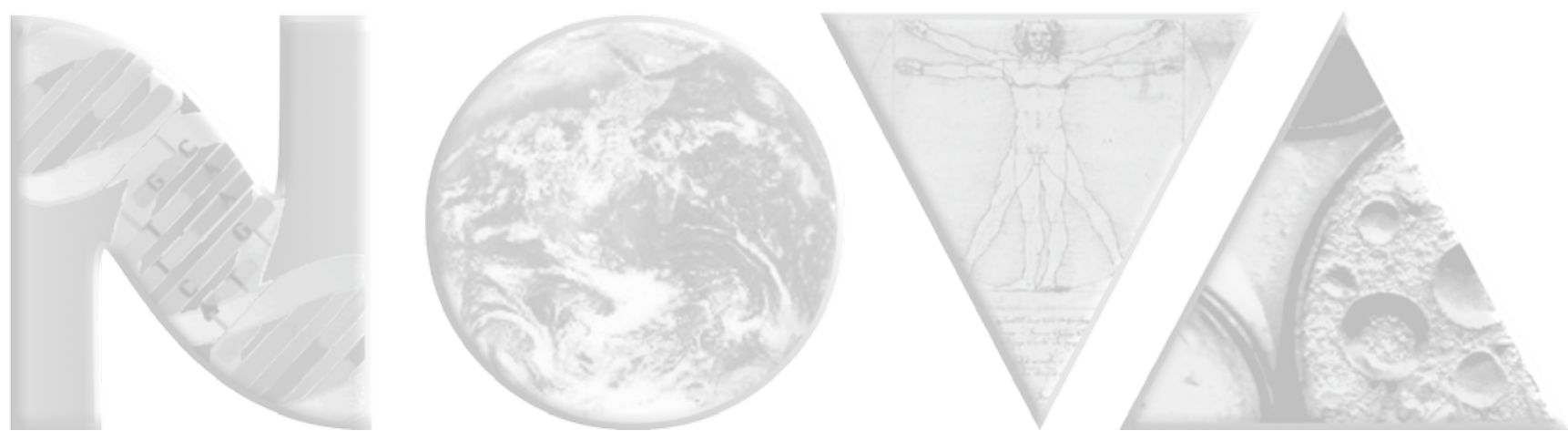